\title{
Aarnoo Prevalência de adiposidade abdominal em adultos de São Francisco do Conde, Bahia, Brasil, 2010*
}

doi: 10.5123/S1679-49742015000100015

\author{
Prevalence of abdominal adiposity in adults in São Francisco do Conde, Bahia, Brazil, 2010
}

\author{
Luciana Carneiro de Oliveira \\ Universidade Estadual de Feira de Santana, Programa de Pós-Graduação em Saúde Coletiva, Feira de Santana-BA, Brasil \\ Luís Eduardo Macedo West \\ Universidade Estadual de Feira de Santana, Departamento de Saúde, Feira de Santana-BA, Brasil \\ Edberig Almeida Araújo \\ Universidade Estadual de Feira de Santana, Departamento de Saúde, Feira de Santana-BA, Brasil \\ Jamyllo Sales Brito \\ Universidade Estadual de Feira de Santana, Departamento de Saúde, Feira de Santana-BA, Brasil \\ Carlito Lopes Nascimento Sobrinho \\ Universidade Estadual de Feira de Santana, Programa de Pós-Graduação em Saúde Coletiva, Feira de Santana-BA, Brasil
}

\section{Resumo}

objetivo: estimar a prevalência de adiposidade abdominal e identificar fatores associados em adultos no município de São Francisco do Conde, estado da Bahia, Brasil. Métodos: estudo transversal com amostra aleatória de 456 indivíduos com idade igual e superior a 18 anos, cadastrados na Estratégia Saúde da Família do município de São Francisco do Conde-BA em 2010; a presença de adiposidade abdominal foi determinada pela aferição da circunferência da cintura. Resultados: a prevalência de adiposidade abdominal foi de $62,1 \%$; nas mulheres, essa prevalência foi de $76,9 \%$ e esteve associada com maior idade (mais de 24 anos) e ter mais de três filhos; nos homens, a prevalência foi de 32,9\%, superior entre aqueles que referiram nunca terem fumado ou serem ex-fumantes; também foi observada associação entre adiposidade abdominal e hipertensão arterial sistêmica, em ambos os sexos. Conclusão: observou-se elevada prevalência de adiposidade abdominal, especialmente em mulheres.

Palavras-chave: Obesidade Abdominal; Circunferência da Cintura; Estudos Transversais.

\begin{abstract}
Objective: to estimate the prevalence of abdominal obesity and to identify associated factors among adults in the municipality of São Francisco do Conde, state of Bahia, Brazil. Methods: this was a cross-sectional study with a random sample of 456 individuals aged 18 years and above, enrolled in the Family Health Program in São Francisco do Conde-BA in 2010; the presence of abdominal adiposity was determined by measuring the waist circumference. Results: overall abdominal obesity prevalence of was $62.1 \%$. It was $76.9 \%$ in women and was associated with increasing age and having more than three children. Prevalence was 32.9\% among men $32.9 \%$ and was and higher former smokers and those who had never smoked. Association between abdominal adiposity and systemic arterial hypertension was observed in both genders. Conclusion: there was high prevalence of abdominal obesity, especially in women.
\end{abstract}

Key words: Obesity, Abdominal; Waist Circumference; Cross-Sectional Studies.

\footnotetext{
* Trabalho elaborado a partir da dissertação de Mestrado 'Prevalência e fatores associados ao excesso de peso em São Francisco do Conde, Bahia', de Luciana Carneiro de Oliveira, defendida junto ao Programa de Pós-Graduação em Saúde Coletiva da Universidade Estadual de Feira de Santana, Feira de Santana-BA, Brasil, em 2014. Financiamento: Fundação de Amparo à Pesquisa do Estado da Bahia (FAPESB) - Termo de Outorga $n^{\circ}$ 0015/2010
}

Endereço para correspondência:

Luciana Carneiro de Oliveira - Avenida Transnordestina, s/n, Novo Horizonte, Feira de Santana-BA, Brasil. CEP:44036-900

E-mail: luacar_oliver@yahoo.com.br 


\section{Introdução}

0 excesso de peso é um importante distúrbio nutricional, tanto nos países desenvolvidos como naqueles em desenvolvimento. ${ }^{1,2}$ Nos países de renda mais elevada, o excesso de peso atinge principalmente a população menos privilegiada, contrapondo-se aos dados de países em desenvolvimento onde a prevalência do excesso de peso é mais relevante na população de maior renda. ${ }^{3}$

No Brasil, desde meados da década de 1970 , inquéritos nutricionais revelaram o crescimento da prevalência de sobrepeso e obesidade nos diversos grupos populacionais, atingindo cerca da metade dos brasileiros a partir de 1989. No entanto, estudos recentes têm apontado maior ocorrência do excesso de peso entre os mais pobres, especialmente entre as mulheres brasileiras. ${ }^{1,4,5}$

Alguns pesquisadores têm se preocupado em caracterizar 0 tipo de obesidade a atingir as populações, uma vez que 0 acúmulo de gordura abdominal oferece maior risco à saúde quando comparado com outras formas de distribuição da gordura no corpo. ${ }^{6,7}$

Medidas antropométricas, entre as quais a circunferência da cintura (CC), vêm sendo utilizadas em estudos populacionais por serem capazes de fornecer estimativas da gordura abdominal, diretamente relacionada à quantidade de tecido adiposo visceral, ${ }^{8-10}$ e por serem reconhecidas como fator de risco para distúrbios metabólicos e doenças cardiovasculares. ${ }^{11}$

\section{0 acúmulo de gordura abdominal oferece maior risco à saúde quando comparado com outras formas de distribuição da gordura no corpo.}

Diversos fatores têm sido apontados como associados ao desenvolvimento de obesidade abdominal, destacando-se os fatores socioeconômicos e os comportamentais. ${ }^{6,12}$ Segundo estudo conduzido por Olinto e colaboradores no estado do Rio Grande do Sul, a baixa escolaridade foi identificada como a variável socioeconômica mais importante. ${ }^{12}$

No Brasil, ainda são poucos os estudos populacionais sobre a caracterização do estado antropométrico em adultos e a avaliação da distribuição de tecido adiposo na região abdominal, ${ }^{6}$ não obstante o padrão de distribuição da gordura corporal possa ter implicações diferenciadas na saúde dos indivíduos.

0 presente estudo objetiva estimar a prevalência de adiposidade abdominal e identificar fatores associados em adultos residentes no município de São Francisco do Conde, estado da Bahia, Brasil.

\section{Métodos}

Trata-se de um estudo transversal de base populacional derivado do projeto de pesquisa intitulado 'Proposta de Vigilância à Saúde para a detecção de distúrbios psíquicos menores e hipertensão arterial em São Francisco do Conde-Bahia-Brasil', realizado em 2010. A pesquisa foi aprovada e financiada pela Fundação de Amparo à Pesquisa do Estado da Bahia (FAPESB), e conduzida por pesquisadores da Sala de Situação e Análise Epidemiológica e Estatística (SSAEE) da Universidade Estadual de Feira de Santana (UEFS), na Bahia, de outubro a dezembro de 2010.

São Francisco do Conde-BA é um município localizado na mesorregião do Recôncavo Baiano, distando 67 $\mathrm{km}$ da capital do estado, Salvador-BA. Em 2010, esse município possuía 31.703 habitantes, distribuídos por sexo em 16.073 mulheres $(50,7 \%)$ e 15.630 homens (49,3\%). São Francisco do Conde-BA apresenta a maior arrecadação per capita entre todos os municípios da América Latina, valores estes oriundos da arrecadação de impostos de uma das maiores refinarias de petróleo do Brasil e única do Norte-Nordeste. 0 município apresentava população predominantemente negra e índice de desenvolvimento humano (IDH) de $0,65 .{ }^{13}$

Foram coletados dados de uma amostra aleatória de indivíduos com idade igual ou superior a 18 anos, cadastrados junto à Estratégia Saúde da Família (ESF) local. 0 tamanho da amostra considerou uma prevalência global da hipertensão arterial no Brasil de 25\% da população adulta, ${ }^{14}$ intervalo de confiança de $95 \%$ (IC95\%) e um erro amostral de 5\%. Foi considerado um efeito de desenho $($ DEFF = design effect $)$ de 1,5 para corrigir o tamanho da amostra, levando-se em conta que a população foi proveniente de várias unidades da ESF. Com essa correção, o tamanho da amostra foi definido em 450 indivíduos com idade igual ou superior a 18 anos $(300 \times 1,5=450)$. Essa amostra permite estimar a prevalência de adiposidade abdominal como resultado deste estudo, com um poder estatístico de $99 \%$. 
Para proceder à seleção dos sujeitos da pesquisa, foi utilizada a técnica de amostragem aleatória estratificada e sistemática. Foram identificadas 29 microáreas cadastradas no Sistema de Informação da Atenção Básica (Siab), da ESF. Cada microárea apresentava aproximadamente 150 famílias e foram sorteadas 10\% do total das famílias cadastradas por microárea. Dessa forma, obteve-se uma amostra com aproximadamente 450 famílias. Afinal, foram sorteadas 456 famílias e de cada família foi selecionado, para entrevista, um indivíduo adulto que atendesse aos critérios de inclusão. Caso o indivíduo sorteado não fosse encontrado após duas visitas ou em caso de óbito do mesmo, fazia-se sua substituição por outro indivíduo da mesma família, buscando-se manter a semelhança em relação a faixa etária e sexo. Caso não fosse possível manter as mesmas características em relação à faixa etária e sexo, outro indivíduo era entrevistado, buscando-se garantir o prazo de 90 dias para a realização da coleta de dados. Foram excluídos do estudo indivíduos acamados, gestantes e portadores de deficiência mental. Foram considerados recusas os indivíduos que decidissem não participar do estudo após a leitura do Termo de Consentimento Livre e Esclarecido.

A coleta de dados foi realizada no período de outubro a dezembro de 2010 , por meio de visitas domiciliares agendadas e realizadas com os participantes em seu respectivo endereço, respeitando-se a distribuição por microárea pertencente à área de abrangência da unidade de Saúde da Família. Entrevistadores (10 estudantes de medicina) previamente treinados aplicaram questionário padronizado contendo informações sobre condições socioeconômicas, demográficas e hábitos de vida, além da aferição de medidas de pressão arterial, peso, estatura e circunferência da cintura.

Com o objetivo de estimar o tempo necessário para aplicação do instrumento, bem como sua clareza e entendimento, foi realizado estudo-piloto em uma microárea pertencente à USF George Américo III, no município de Feira de Santana-BA. A coleta de dados foi iniciada após os ajustes sugeridos pelo estudo-piloto.

A medida da circunferência da cintura foi realizada com fita métrica inelástica (confeccionada em material de fibra de vidro, ou fiberglass), com capacidade de até $150 \mathrm{~cm}$ e grau de precisão de $1 \mathrm{~cm}$. Os indivíduos foram medidos descalços, em posição ereta, braços ao longo do corpo, pés juntos, com o olhar voltado para o horizonte, com o peso dividido entre ambas as pernas, posicionados com os pés unidos e contra uma superfície plana (parede). A medida da CC foi tomada no ponto médio da distância entre a borda inferior do gradil costal e o ilíaco, no plano horizontal. ${ }^{15} 0 \mathrm{~s}$ pontos de corte dessa medida estabelecem o grau de risco para doenças crônicas não-transmissíveis. A circunferência da cintura foi categorizada utilizando-se os seguintes pontos de corte: normal ( $<80 \mathrm{~cm}$ para mulheres; e $<94 \mathrm{~cm}$ para homens); de risco elevado $(80 \geq \mathrm{CC}<88 \mathrm{~cm}$ para mulheres; e $94 \geq \mathrm{CC}<102 \mathrm{~cm}$ para homens); e de risco muito elevado ( $\geq 88 \mathrm{~cm}$ para mulheres; $\mathrm{e} \geq 102 \mathrm{~cm}$ para homens). ${ }^{16}$ Para o presente estudo, utilizou-se pontos de corte da presença de adiposidade abdominal: nas mulheres, de $\mathrm{CC} \geq 80 \mathrm{~cm}$; e entre os homens, de $\mathrm{CC} \geq 94 \mathrm{~cm}$. A padronização e a aferição das medidas antropométricas foram realizadas dentro das recomendações do Departamento de Nutrição da Universidade de São Paulo. ${ }^{17}$

As variáveis socioeconômicas e demográficas estudadas foram:

- idade em anos completos no momento da entrevista, posteriormente categorizada em seis estratos: 18-24, 25-34, 35-44, 45-54, 55-64 e 65 ou mais anos;

- renda familiar per capita, de acordo com informação dos componentes da família, estratificada segundo a renda mensal familiar em salários mínimos (SM) - menor que um ( $<1 \mathrm{SM})$, entre 1 e 2 (1-2 SM) e igual ou maior que 3 salários mínimos ( $\geq 3$ $\mathrm{SM})$-, sendo o valor do salário mínimo vigente, à época do estudo, de $\mathbf{R} \$ 510,00$;

- escolaridade, coletada em nível de estudo e categorizada como Analfabeto a Ensino Fundamental incompleto, Ensino Fundamental completo a Ensino Médio incompleto e Ensino Médio completo a Ensino Superior; e

- situação conjugal, dividida em duas categorias: solteiro/separado/viúvo; e casado/união estável.

Em relação aos hábitos de vida, a variável tabagismo foi categorizada de acordo com o uso referido: não fuma, fuma diariamente e ex-fumante.

A variável relacionada à prática de atividade física foi dicotomizada em 'sim' ou 'não', e a respectiva frequência foi dividida em três estratos: pratica uma vez na semana, pratica 2 a 4 vezes na semana e mais de 4 vezes na semana.

A variável hipertensão arterial também foi dicotomizada em 'sim' ou 'não', segundo a resposta do 
entrevistado (morbidade referida) e/ou o resultado da medida de pressão arterial (PA): $\geq 140 \mathrm{mmHg}$ para PA sistólica; e/ou $\geq 90 \mathrm{mmHg}$ para a PA diastólica. No presente estudo, não foi investigado o uso de medicamentos anti-hipertensivos.

Com o intuito de detectar possíveis erros de digitação, foram construídos dois bancos de dados pelo programa EpiData versão 3.1. Após a verificação de erros e inconsistências, a análise dos dados utilizou-se dos programas Statistical Package for the Social Sciences (SPSS ${ }^{\circledR}$ ) versão 9.0 for Windows ${ }^{18} \mathrm{e}$ OpenEpi versão 3.03 .

A adiposidade abdominal foi considerada como variável-desfecho. Foram calculadas as frequências absolutas e relativas das variáveis qualitativas e as medidas de tendência central e de dispersão das variáveis numéricas. Realizou-se a análise bivariada entre as variáveis sociodemográficas, socioeconômicas, hábitos de vida e estado nutricional por meio do teste de $\mathrm{x}^{2}$ (qui-quadrado) de Pearson e do teste exato de Fisher. Outrossim, foram calculadas as razões de prevalência (RP) e respectivos intervalos de confiança de $95 \%$. Nas análises, considerou-se 0 nível de significância estatística de 5\%.

0 estudo foi aprovado pela Secretaria Municipal de Saúde de São Francisco do Conde-BA e pelo Comitê de Ética em Pesquisa (CEP) da Universidade Estadual de Feira de Santana (UEFS): Certificado de Apresentação para Apreciação Ética (CAAE) $\mathrm{n}^{0}$ 0008.0.059, em conformidade com a Resolução do Conselho Nacional de Saúde (CNS) nº 466, de 12 de dezembro de 2012.

\section{Resultados}

Dos 456 indivíduos inicialmente elegíveis para o estudo, $72(15,8 \%)$ não foram encontrados no momento da visita, sendo substituídos com base nos critérios adotados pelo estudo. Não houve recusas. A maioria dos participantes foi do sexo feminino $(66,2 \%)$ e sua média de idade foi de 43,2 anos (desvio-padrão: 15,6). A distribuição por sexo das características sociodemográficas e socioeconômicas e hábitos de vida da população avaliada consta na Tabela 1. A maior parte da amostra estudada encontrava-se nas faixas etárias de 25-34 (25,9\%) e 35-44 anos (21,1\%). Observou-se maior número de indivíduos casados, com atividade remunerada e renda mensal de 1 a 2 salários mínimos.
A prevalência de adiposidade abdominal nos adultos de São Francisco do Conde-BA foi de $62,1 \%$, sendo de $76,9 \%$ para o sexo feminino e de $32,9 \%$ para 0 sexo masculino, especialmente no subgrupo de risco substancialmente aumentado (Tabela 1).

No sexo masculino, observou-se maior prevalência de adiposidade abdominal entre os indivíduos na faixa etária de 35 a 44 anos, ex-fumantes ou que indicaram nunca terem fumado, e com hipertensão arterial sistêmica referida $\left(1,75 ; \mathrm{IC}_{95 \%}: 1,12-2,71\right)$ (Tabela 2$)$.

No sexo feminino, a maior prevalência de adiposidade abdominal ocorreu na faixa etária de 55 a 64 anos; mulheres mais jovens (18-24 anos) apresentaram menor prevalência de adiposidade abdominal $\left(0,56 ; \mathrm{IC}_{95 \%}: 0,35-0,89\right)$ do que aquelas com mais de 65 anos. As mulheres que informaram ter mais de 3 filhos apresentaram maior prevalência do desfecho. Entre aquelas que referiram hipertensão arterial, a prevalência do desfecho foi 1,24 ( $\left.\mathrm{IC}_{95 \%}: 1,10-1,39\right)$ vezes superior quando comparada com as respectivas prevalências observadas em mulheres que não referiram a morbidade (Tabela 3).

\section{Discussão}

Verificou-se elevada prevalência de adiposidade abdominal entre os indivíduos estudados, principalmente quando mulheres. Essa maior ocorrência esteve associada com o (i) aumento da faixa etária e (ii) ter mais de três filhos. Para os homens, a prevalência de adiposidade abdominal foi superior entre aqueles que referiram nunca terem fumado ou serem ex-fumantes. Observou-se também, em ambos os sexos, associação entre adiposidade abdominal e hipertensão arterial sistêmica.

Prevalência elevada de adiposidade abdominal $(51,9 \%)$ também foi observada em adultos residentes no estado de Pernambuco, graças a estudo conduzido por Pinho e colaboradores. ${ }^{19}$ No entanto, outros estudos populacionais realizados na região Nordeste, como em Salvador-BA ${ }^{20}$ e no estado do Maranhão, ${ }^{21}$ obtiveram prevalências inferiores, de $28,1 \%$ e $46,3 \%$ respectivamente. A maior prevalência encontrada no presente estudo pode estar relacionada à composição da amostra do estudo: indivíduos com baixo nível socioeconômico (baixa renda e baixa escolaridade). Normalmente, esse grupo apresenta maior consumo de alimentos ricos em gorduras e açúcares - mais 
Tabela 1 - Caracterização da amostra estudada segundo sexo no município de São Francisco do Conde, Bahia, 2010

\begin{tabular}{|c|c|c|c|c|c|c|}
\hline \multirow{2}{*}{ Variáveis } & \multicolumn{2}{|c|}{ Total } & \multicolumn{2}{|c|}{ Feminino } & \multicolumn{2}{|c|}{ Masculino } \\
\hline & $(N=456)$ & $\%$ & $(n=302)$ & $\%$ & $(n=154)$ & $\%$ \\
\hline \multicolumn{7}{|l|}{ Circunferência da cintura } \\
\hline Normal & 171 & 37,9 & 69 & 23,1 & 102 & 67,1 \\
\hline Risco elevado & 86 & 19,1 & 62 & 20,7 & 24 & 15,8 \\
\hline Risco muito elevado & 194 & 43,0 & 168 & 56,2 & 26 & 17,1 \\
\hline \multicolumn{7}{|l|}{ Idade (em anos) } \\
\hline $18-24$ & 46 & 10,1 & 28 & 9,3 & 18 & 11,7 \\
\hline $25-34$ & 118 & 25,9 & 84 & 27,9 & 34 & 22,1 \\
\hline $35-44$ & 96 & 21,1 & 59 & 19,6 & 37 & 24,0 \\
\hline $45-54$ & 78 & 17,1 & 55 & 18,3 & 23 & 14,9 \\
\hline $55-64$ & 69 & 15,2 & 47 & 15,6 & 22 & 14,3 \\
\hline$\geq 65$ & 48 & 10,5 & 28 & 9,3 & 20 & 13,0 \\
\hline \multicolumn{7}{|l|}{ Situação conjugal } \\
\hline Casado & 265 & 58,2 & 168 & 55,8 & 97 & 63,0 \\
\hline Solteiro & 190 & 41,8 & 133 & 44,2 & 57 & 37,0 \\
\hline \multicolumn{7}{|l|}{ Escolaridade } \\
\hline Analfabeto a Ensino Fundamental incompleto & 232 & 51,1 & 149 & 49,5 & 83 & 54,2 \\
\hline Ensino Fundamental completo a Ensino Médio incompleto & 72 & 15,9 & 41 & 13,6 & 31 & 20,3 \\
\hline Ensino Médio completo a Ensino Superior & 150 & 33,0 & 111 & 36,9 & 39 & 25,5 \\
\hline \multicolumn{7}{|l|}{ Renda familiar (em salários mínimos) } \\
\hline$<1$ & 82 & 18,5 & 37 & 12,5 & 45 & 30,4 \\
\hline $1-2$ & 300 & 67,6 & 214 & 72,3 & 86 & 58,1 \\
\hline$\geq 3$ & 62 & 14,0 & 45 & 15,2 & 17 & 11,5 \\
\hline \multicolumn{7}{|l|}{ Número de filhos } \\
\hline Nenhum & 76 & 16,7 & 34 & 11,3 & 42 & 27,3 \\
\hline 1 ou 2 & 173 & 37,9 & 127 & 42,1 & 46 & 29,9 \\
\hline$\geq 3$ & 207 & 45,4 & 141 & 46,7 & 66 & 14,5 \\
\hline \multicolumn{7}{|l|}{ Tabagismo } \\
\hline Nunca fumou & 290 & 64,6 & 208 & 70,0 & 82 & 53,9 \\
\hline Ex-fumante & 111 & 24,7 & 67 & 22,6 & 44 & 28,9 \\
\hline Fuma diariamente & 48 & 10,7 & 22 & 7,4 & 26 & 17,1 \\
\hline \multicolumn{7}{|l|}{ Prática de atividade física } \\
\hline Sim & 138 & 30,3 & 62 & 20,5 & 76 & 49,4 \\
\hline Não & 318 & 69,7 & 240 & 79,5 & 78 & 50,6 \\
\hline \multicolumn{7}{|l|}{ Frequência de atividade física } \\
\hline $1 \mathrm{vez} /$ semana & 23 & 16,8 & 3 & 4,8 & 20 & 26,7 \\
\hline $2-4$ vezes/semana & 57 & 41,6 & 28 & 45,2 & 29 & 38,7 \\
\hline$>4$ vezes/semana & 57 & 41,6 & 31 & 50,0 & 26 & 34,7 \\
\hline \multicolumn{7}{|l|}{ Hipertensão autorreferida } \\
\hline Sim & 155 & 34,0 & 112 & 37,1 & 43 & 27,9 \\
\hline Não & 278 & 61,0 & 181 & 59,9 & 97 & 63,0 \\
\hline Não sabe & 23 & 5,0 & 9 & 3,0 & 14 & 9,1 \\
\hline
\end{tabular}

Nota: dados válidos, excluídos os ignorados. 
Tabela 2 - Prevalência de adiposidade abdominal segundo característica socioeconômica, hábitos de vida e hipertensão autorreferida da população masculina adulta $\geq 18$ anos de idade $(n=456)$ no município São Francisco do Conde, Bahia, 2010

\begin{tabular}{|c|c|c|c|c|c|c|c|}
\hline \multirow{2}{*}{ Variáveis } & \multicolumn{5}{|c|}{ Adiposidade abdominal } & \multirow{2}{*}{$\mathrm{RP}^{\mathrm{b}}\left(\mathrm{IC}_{95 \%}{ }^{\mathrm{c}}\right)$} & \multirow{2}{*}{$\begin{array}{l}\text { Valor } \\
\text { dep }\end{array}$} \\
\hline & $\mathrm{N}^{\mathrm{a}}$ & Presente & $\%$ & Ausente & $\%$ & & \\
\hline Idade (em anos) & 152 & & & & & & \\
\hline $18-24$ & & 2 & 11,1 & 16 & 88,9 & $0,33(0,07-1,43)$ & $0,228^{e}$ \\
\hline $25-34$ & & 7 & 20,6 & 27 & 79,4 & $0,61(0,24-1,56)$ & $0,314^{d}$ \\
\hline $35-44$ & & 18 & 48,6 & 19 & 51,4 & $1,45(0,70-3,03)$ & $0,283^{d}$ \\
\hline $45-54$ & & 10 & 43,4 & 13 & 56,5 & $1,30(0,58-2,91)$ & $0,508^{d}$ \\
\hline $55-64$ & & 7 & 31,8 & 15 & 68,2 & $0,95(0,39-2,33)$ & $0,918^{d}$ \\
\hline$\geq 65$ & & 6 & 33,3 & 12 & 66,7 & 1,00 & \\
\hline Situação conjugal & 152 & & & & & & \\
\hline Casado & & 35 & 36,4 & 61 & 63,5 & $1,36(0,81-2,26)$ & $0,221^{d}$ \\
\hline Solteiro & & 15 & 26,7 & 41 & 73,2 & 1,00 & \\
\hline Escolaridade & 151 & & & & & & \\
\hline Analfabeto a Ensino Fundamental incompleto & & 17 & 33,3 & 54 & 66,7 & $0,71(0,39-1,31)$ & $0,-0,291^{d}$ \\
\hline Ensino Fundamental completo a Ensino Médio incompleto & & 9 & 29,0 & 22 & 71,0 & $0,87(0,42-1,76)$ & $0,700^{d}$ \\
\hline Ensino Médio completo aEnsino Superior & & 13 & 33,3 & 26 & 66,7 & 1,00 & \\
\hline Renda familiar (em salários mínimos) & 146 & & & & & & \\
\hline$<1$ & & 22 & 48,9 & 23 & 51,1 & $1,18(0,62-2,25)$ & $0,0587^{d}$ \\
\hline $1-2$ & & 20 & 23,7 & 64 & 76,2 & $0,57(0,29-1,14)$ & $0,140^{d}$ \\
\hline$\geq 3$ & & 7 & 41,2 & 10 & 58,8 & 1,00 & \\
\hline Paridade (no de filhos) & 127 & & & & & & \\
\hline Nenhum & & 12 & 28,5 & 30 & 71,4 & 1,00 & \\
\hline 1 ou 2 & & 13 & 28,2 & 33 & 71,7 & $0,98(0,50-1,92)$ & $0,974^{d}$ \\
\hline$\geq 3$ & & 25 & 39,1 & 39 & 60,9 & $1,36(0,77-2,41)$ & $0,268^{d}$ \\
\hline Tabagismo & 150 & & & & & & \\
\hline Nunca fumou & & 30 & 36,6 & 52 & 63,4 & $3,04(1,01-9,15)$ & $0,029^{e}$ \\
\hline Ex-fumante & & 16 & 37,2 & 27 & 62,8 & $3,10(1,00-9,60)$ & $0,044^{e}$ \\
\hline Fuma diariamente & & 3 & 12,0 & 22 & 88,0 & 1,00 & \\
\hline Prática de atividade física & 152 & & & & & & \\
\hline Sim & & 27 & 35,5 & 49 & 64,5 & 1,00 & \\
\hline Não & & 23 & 30,3 & 53 & 69,7 & $0,85(0,53-1,34)$ & $0,489^{d}$ \\
\hline Frequência de atividade física & 75 & & & & & & \\
\hline $1 \mathrm{vez} /$ semana & & 5 & 25,0 & 15 & 75,0 & $0,50(0,21-1,17)$ & $0,150^{\mathrm{e}}$ \\
\hline $2-4$ vezes/semana & & 9 & 31,0 & 20 & 69,0 & $0,62(0,31-1,20)$ & $0,151^{d}$ \\
\hline$>4$ vezes/semana & & 13 & 50,0 & 13 & 50,0 & 1,00 & \\
\hline Hipertensão autorreferida & 152 & & & & & & \\
\hline $\operatorname{Sim}$ & & 20 & 47,7 & 22 & 52,4 & $1,75(1,12-2,71)$ & $0,016^{d}$ \\
\hline Não & & 30 & 23,3 & 80 & 72,7 & 1,00 & \\
\hline
\end{tabular}

a) Respostas válidas, excluídas as ignoradas.

b) RP: razão de prevalência

c) IC : intervalo de confiança de $95 \%$

d) Teste do qui-quadrado de Pearson

e) Teste exato de Fisher 
Tabela 3 - Prevalência de adiposidade abdominal segundo características socioeconômicas, hábitos de vida e hipertensão autorreferida da população feminina adulta $\geq 18$ anos de idade $(n=456)$ no município de São Francisco do Conde, Bahia, 2010

\begin{tabular}{|c|c|c|c|c|c|c|c|}
\hline \multirow{2}{*}{ Variáveis } & \multicolumn{5}{|c|}{ Adiposidade abdominal } & \multirow{2}{*}{$\operatorname{RP}^{b}\left(\mathrm{IC}_{95 \%}\right)^{\mathrm{c}}$} & \multirow{2}{*}{$\underset{p^{d}}{\text { Valor de }}$} \\
\hline & $\mathrm{N}^{\mathrm{a}}$ & Presente & $\%$ & Ausente & e $\%$ & & \\
\hline Idade (em anos) & 298 & & & & & & \\
\hline $18-24$ & & 12 & 44,4 & 15 & 55,6 & $0,56(0,35-0,89)$ & $0,009^{d}$ \\
\hline $25-34$ & & 59 & 71,1 & 24 & 28,9 & $0,90(0,71-1,14)$ & $0,440^{d}$ \\
\hline $35-44$ & & 50 & 84,7 & 9 & 15,3 & $1,10(0,86-1,31)$ & $0,476^{\mathrm{d}}$ \\
\hline $45-54$ & & 46 & 83,6 & 9 & 16,4 & $1,10(0,84-1,33)$ & $0,570^{d}$ \\
\hline $55-64$ & & 40 & 86,9 & 6 & 13,0 & $1,10(0,88-1,38)$ & $0,342^{d}$ \\
\hline$\geq 65$ & & 22 & 78,6 & 6 & 21,4 & 1,00 & \\
\hline Situação conjugal & 298 & & & & & & \\
\hline Casado & & 133 & 79,7 & 34 & 20,4 & $1,10(0,95-1,23)$ & $0,196^{d}$ \\
\hline Solteiro & & 96 & 73,2 & 35 & 26,7 & 1,00 & \\
\hline Escolaridade & 298 & & & & & & \\
\hline Analfabeto a Ensino Fundamental incompleto & & 120 & 81,1 & 28 & 18,9 & $1,13(0,98-1,30)$ & $0,072^{d}$ \\
\hline Ensino fundamental completo a Ensino Médio incompleto & & 32 & 78,1 & 9 & 22,0 & $1,10(0,89-1,33)$ & $0,423^{d}$ \\
\hline Ensino Médio completo a Ensino Superior & & 78 & 71,5 & 31 & 28,4 & 1,00 & \\
\hline Renda familiar (em salários mínimos) & 293 & & & & & & \\
\hline$<1$ & & 28 & 77,8 & 8 & 22,2 & $1,00(0,79-1,26)$ & $0,999^{d}$ \\
\hline $1-2$ & & 163 & 76,9 & 49 & 23,1 & $0,98(0,83-1,17)$ & $0,897^{d}$ \\
\hline$\geq 3$ & & 35 & 77,8 & 10 & 22,2 & 1,00 & \\
\hline Número de filhos & 299 & & & & & & \\
\hline Nenhum & & 18 & 54,6 & 15 & 45,5 & $0,28(0,19-0,43)$ & $<0,001^{\mathrm{d}}$ \\
\hline 1 ou 2 & & 91 & 72,8 & 34 & 27,2 & $0,84(0,74-0,96)$ & $0,008^{d}$ \\
\hline$\geq 3$ & & 121 & 85,9 & 20 & 14,2 & 1,00 & \\
\hline Tabagismo & 294 & & & & & & \\
\hline Nunca fumou & & 156 & 76,1 & 49 & 23,9 & $1,04(0,80-1,36)$ & $0,725^{d}$ \\
\hline Ex-fumante & & 55 & 82,1 & 12 & 17,9 & $1,12(0,85-1,49)$ & $0,342^{\mathrm{d}}$ \\
\hline Fuma diariamente & & 16 & 72,7 & 6 & 27,3 & 1,00 & \\
\hline Prática de atividade física & 299 & & & & & & \\
\hline Sim & & 48 & 77,4 & 14 & 22,6 & 1,00 & \\
\hline Não & & 182 & 76,8 & 55 & 23,2 & $0,99(0,85-1,15)$ & $0,917^{d}$ \\
\hline Frequência de atividade física & 62 & & & & & & \\
\hline $1 \mathrm{vez} /$ semana & & 2 & 66,6 & 1 & 33,3 & $0,82(0,36-1,87)$ & $0,999^{e}$ \\
\hline $2-4$ vezes/semana & & 21 & 75,0 & 7 & 25,0 & $0,93(0,70-1,22)$ & \\
\hline$>4$ vezes/semana & & 25 & 80,7 & 6 & 19,4 & 1,00 & $0,601^{d}$ \\
\hline Hipertensão autorreferida & 299 & & & & & & \\
\hline Sim & & 98 & 87,5 & 14 & 12,5 & $1,24(1,10-1,39)$ & $<0,001^{d}$ \\
\hline Não & & 132 & 70,6 & 55 & 29,4 & 1,00 & \\
\hline
\end{tabular}

a) Respostas válidas, excluídas as ignoradas.

b) RP: razão de prevalência

c) IC : intervalo de confiança de $95 \%$

d) Teste do qui-quadrado de Pearson

e) Teste exato de Fisher 
palatáveis e baratos - e pobres em proteínas - em geral, mais caros. ${ }^{4}$

No presente estudo, o fato de as mulheres apresentarem maior prevalência de adiposidade abdominal corrobora os resultados de outras pesquisas. . $92,21,22^{2}$ Uma possível explicação para esses achados pode estar relacionada às gestações, à ação dos hormônios femininos e seu impacto sobre o armazenamento de gorduras no corpo. ${ }^{23}$

A paridade tem sido associada à presença de adiposidade abdominal. ${ }^{20,23} 0$ estudo em tela constatou que as mulheres com mais de 3 filhos apresentaram maior prevalência do desfecho. As alterações hormonais experimentadas pelas mulheres, decorrentes do período gravídico-puerperal, estão relacionadas ao aumento da deposição de gordura na região abdominal. ${ }^{23}$ Ademais, o estiramento da musculatura abdominal resultante das gestações, fenômeno indispensável ao crescimento uterino, leva à separação dos feixes dos músculos retos abdominais e portanto, favorece a obesidade abdominal. ${ }^{24}$

A prevalência de adiposidade abdominal também se apresentou elevada entre os adultos jovens, mesmo tendo aumentado com o crescimento da faixa etária, corroborando o resultado de estudo realizado em Teresina, estado do Piaui. ${ }^{25}$ Tal situação pode estar relacionada à ingestão de alimentos ricos em gorduras e açúcares e pobres em fibras, típicos da dieta ocidental, adotada pelas sociedades contemporâneas em nível global. ${ }^{4}$

Não fumantes e ex-fumantes apresentaram maiores prevalências de adiposidade abdominal do que os fumantes, o que corrobora resultados de outros estudos. ${ }^{19,20,23}$ A maioria das pesquisas mostra que a suspensão do hábito de fumar parece resultar em ganho ponderal, principalmente pelo aumento da ingestão calórica, destacando-se o consumo de alimentos ricos em açúcares e gorduras, a que se soma o fato de a suspensão do tabagismo acarretar uma diminuição da taxa metabólica basal, que pode variar entre 4 e 16\% - 0 que representaria menos de $40 \%$ do ganho ponderal. ${ }^{26}$ No entanto, um resultado como esse deve ser analisado cuidadosamente, pois, como salientam Sousa e colaboradores ${ }^{27}$ mesmo que o hábito de fumar exerça efeito sobre o ganho de peso, a adoção de estilo de vida mais saudável garante maiores benefícios aos indivíduos.

A disposição da gordura na região abdominal é reconhecida como fator de risco para distúrbios me- tabólicos e doenças cardiovasculares. ${ }^{28,29}$ Neste estudo, foram observadas maiores prevalências de adiposidade abdominal entre os indivíduos que referiram hipertensão arterial embora, aqui, não se tenha investigado 0 uso de medicamentos anti-hipertensivos, o que pode ter levado a um viés de aferição.

0 delineamento transversal desta pesquisa constitui uma das limitações ao desenvolvimento de análises das relações entre as variáveis preditoras (características socioeconômicas, demográficas e hábitos de vida) e 0 desfecho (adiposidade abdominal) por não ser possível estabelecer um nexo causal, dada a possibilidade de ocorrência do viés de causalidade reversa. Além disso, não foram realizadas análises ajustadas que permitissem o controle sobre potenciais fatores de confusão, tampouco o teste de interações, o que indica a necessidade de manter cautela na interpretação dos resultados encontrados..$^{30}$

Outra limitação deste estudo refere-se à possibilidade de ocorrência de viés de seleção, uma vez que a maioria das pessoas entrevistadas nas visitas domiciliares foi de mulheres $(66,2 \%)$, situação inalterada mesmo quando realizadas visitas de reposição em horários alternativos com o propósito de minimizar a perda de indivíduos do sexo masculino.

Um aspecto importante a ser discutido refere-se ao ponto de corte adotado para determinar a adiposidade abdominal $(\geq 80 \mathrm{~cm}$ para mulheres; $\geq 94 \mathrm{~cm}$ para homens), também utilizado em outros estudos,,$^{20,23}$ que garante maior preditividade em relação a outros pontos de corte. Baixos valores da medida da circunferência abdominal (baixa adiposidade abdominal) já configuram risco para as doenças cardiovasculares. ${ }^{19}$

Por meio de um estudo transversal de base populacional e posterior análise, foi possível estimar a prevalência de adiposidade abdominal e identificar fatores associados em adultos de São Francisco do Conde-BA. A compreensão de como a adiposidade abdominal encontra-se distribuída na população e seus fatores associados aponta para a necessidade do planejamento e implementação de ações voltadas ao controle e prevenção desse relevante problema de saúde. Recomenda-se a inclusão dessas ações nas atividades de responsabilidade dos agentes comunitários de saúde vinculados às unidades de Saúde da Família, pelo rastreamento da adiposidade abdominal com a aferição da circunferência da cintura na população 
adscrita à ESF. A implementação de atividade física em praças públicas e centros esportivos, a divulgação de informações relativas aos fatores de risco e às consequências do excesso de peso, bem como a realização de ações intersetoriais visando ações políticas e sociais de maior amplitude, são medidas importantes na prevenção e no controle da adiposidade abdominal.

\section{Contribuição dos autores}

\section{Referências}

1. Pinheiro ARO, Freitas SFT, Corso ACT. Uma abordagem epidemiológica da obesidade. Rev Nutr. 2004 out-dez;17(4):523-33.

2. Tardido AP, Falcão MC. 0 impacto da modernização na transição nutricional e obesidade. Rev Bras Nutr Clin. 2006;21(2):117-24.

3. Al-Shammari AS, Khoja T, Gad A. Community based study of obesity among children and adults in Ryadh, Saudi Arabia. Food Nutr Bull. 2001 jun;22(2):178-83.

4. Instituto Brasileiro de Geografia e Estatística. Pesquisa de Orçamentos Familiares 2008-2009: antropometria e estado nutricional de crianças, adolescentes e adultos no Brasil [Internet]. Rio de Janeiro: Instituto Brasileiro de Geografia e Estatística; 2010 [citado 2013 out 23]. Disponível em: http://www.ibge.gov. br/home/estatistica/populacao/condicaodevida/ pof/2008_2009_encaa/pof_20082009_encaa.pdf

5. Gigante DP, Moura EC, Sardinha LMV. Prevalência de excesso de peso e obesidade e fatores associados, Brasil, 2006. Rev Saude Publica. 2009 nov;43(2):83-9.

6. Castanheira M, Olinto MTA, Gigante DP. Associação de variáveis sócio-demográficas e comportamentais com a gordura abdominal em adultos: estudo de base populacional no Sul do Brasil. Cad Saude Publica. 2003;19 supl 1:S55-S65.

7. Damascena LL, Pereira Neto N, Pereira VA. Correlação entre obesidade abdominal, IMC e risco cardiovascular. In: $11^{\circ}$ Encontro de Iniciação à Docência. 2008 abr 9-11; João Pessoa: Universidade Federal da Paraíba; 2008.

8. Pouliot MC, Després JP, Lemieux S, Moorjani S, Bourchard C, Tremblay A, et al. Waist circumference and abdominal sagittal diameter: best simple anthropometric indexes of abdominal visceral adipose
Oliveira LC e Nascimento Sobrinho CL contribuíram na concepção e delineamento do estudo, análise e interpretação dos resultados, redação e revisão crítica do conteúdo intelectual.

Araújo EA, Brito JS e West LEM participaram do delineamento do estudo, análise e interpretação dos resultados.

Todos os autores aprovaram a versão final do manuscrito e declaram serem responsáveis por todos os aspectos do trabalho, garantindo sua precisão e integridade.

tissue accumulation and related cardiovascular risk in men and women. Am J Cardiol. 1994 Mar;73(7):460-8.

9. Clasey JL, Bouchard C, Teates CD, Riblett JE, Thorner MO, Hartman ML, et al. The use of anthropometric and dual-energy X-ray absorptiometry (DXA) measures to estimative total abdominal and abdominal visceral fat in men and women. Obes Res. 1999 May;7(3):256-64.

10. Pitanga FJG. Antropometria na avaliação da obesidade abdominal e risco coronariano. Rev Bras Cineantropom Desempenho Hum. 2011 mai-jun;13(3):238-41.

11. Dyer AR, Liu K, Walsh M, Kiefe C, Jacobs Jr DR, Bild DE. Ten-year incidencof elevated blood pressure and its predictors: the CARDIA study. J Hum Hypertens. 1999 Jan;13(1):13-21.

12. Olinto MTA, Nácul LC, Dias-da-Costa JS, Gigante DP, Menezes AMB, Macedo S. Níveis de intervenção para obesidade abdominal: prevalência e fatores associados. Cad Saude Publica. 2006 jun;22(6):1207-15.

13. Instituto Brasileiro de Geografia e Estatística. Resultados do Censo Demográfico, 2010: Bahia [Internet]. Rio de Janeiro: Instituto Brasileiro de Geografia e Estatística; 2010 [citado 2013 out 23]. Disponível em: http://www.ibge.gov.br/home/ estatistica/populacao/censo2010/tabelas_pdf/total_ populacao_bahia.pdf

14. Ministério da Saúde (BR). Hipertensão arterial sistêmica para o SUS. Brasília: Ministério da Saúde; 2006. (Cadernos de atenção básica; n. 15)

15. Habicht JP, Butz WP. Measurement of health and nutrition effects of large-scale nutrition intervention projects. In: Klein RE. Evaluation of the impact of nutrition and health programs. New York: Plenum Press; 1979. p. 133-89. 
16. World Health Organization. Obesity: preventing and managing the global epidemic. In: Report of a WHO Consultation on Obesity; 1997 Jun 3-5; Geneva. Geneva: World Health Organization; 1998.

17. Universidade de São Paulo. Faculdade de Saúde Pública. Laboratório de Avaliação Nutricional de Populações. Manual de técnicas antropométricas: guia para realização de medidas antropométricas [Internet]. São Paulo: Lanpop; 2010 [citado 2010 jun 10]. Disponível em: http://www.fsp.usp.br/lanpop/ index_arquivos/Page 571.htm

18. SPSS Inc. SPSS Base 9.0: applications guide. Chicago: SPSS Inc; 1999.

19. Pinho CPS, Diniz AS, Arruda IKG, Batista Filho M, Coelho PC, Sequeira LAS, et al. Prevalência e fatores associados à obesidade abdominal em indivíduos na faixa etária de 25 a 59 anos do Estado de Pernambuco, Brasil. Cad Saude Publica. 2013 fev;29(2):313-24.

20. Oliveira LPM, Assis AMO, Silva MCM, Santana MLP, Santos NS, Pinheiro SMC, et al. Fatores associados a excesso de peso e concentração de gordura abdominal em adultos na cidade de Salvador, Bahia, Brasil. Cad Saude Publica. 2009 mar;25(3):570-82.

21. Veloso HJF, Silva AAM. Prevalência e fatores associados à obesidade abdominal e ao excesso de peso em adultos maranhenses. Rev Bras Epidemiol. 2010 set;13(3):400-12.

22. Pimenta AM, Kac G, Gazzinelli A, Correa-Oliveira R, Velásquez-Meléndez G. Associação entre obesidade central, triglicerídeos e hipertensão arterial em uma área rural do Brasil. Arq Bras Cardiol. 2008 jun;90(6):419-25.
23. Ronsoni RM, Coutinho MSSA, Pereira MR, Silva RH, Becker IC, Sehnen Jr L. Prevalência de obesidade e seus fatores associados na população de Tubarão SC. ACM Arq Catarin Med. 2005 jul-set;34(3):51-7.

24. Polden M, Mantle J. Fisioterapia em ginecologia e obstetrícia. 2. ed. São Paulo: Santos; 2000.

25. Holanda LGM, Martins MCC, Souza Filho MD, Carvalho CMRG, Assis RC, Leal LMM, et al. Excesso de peso e adiposidade central em adultos de Teresina-PI. Rev Assoc Med Bras. 2011 jan-fev;57(1):50-5.

26. Filozof C, Fernadez Pinilla MC, Fernandez-Cruz A. Smoking cessation and weight gain. Obes Rev. 2004 May;5(2):95-103.

27. Sousa RMRP, Sobral DP, Paz SMRS, Martins MCC. Prevalência de sobrepeso e obesidade entre funcionários plantonistas de unidades de saúde de Teresina-PI. Rev Nutr. 2007 set-out;20(5):473-82.

28. Martins IS, Marinho SP. O potencial diagnóstico dos indicadores da obesidade centralizada. Rev Saude Publica. 2003 dez;37(6):760-7.

29. Gus M, Fuchs SC, Moreira LB, Moraes RS, Wiehe M, Silva AF, et al. Association between different measurements of obesity and the incidence of hypertension. Am J Hypertens. 2004 Jan;17(1):50-3.

30. Pereira MG. Epidemiologia teoria e prática. Rio de Janeiro: Guanabara Koogan; 2005.

Recebido em 18/04/2014

Aprovado em 20/11/2014 